\title{
Aprendizaje móvil en Educación Física. Una propuesta de innovación en ESO
}

\author{
M-Learning in Physical Education. An innovation proposal in Secondary \\ Education
}

\author{
Alejandro Martínez Martínez \\ Universidad de Murcia, España (alejandroffx@hotmail.com)
}

Recibido el 17 de julio de 2018; revisado el 5 de octubre de 2018; aceptado el 5 de julio de 2019; publicado el 1 de diciembre de 2019

\section{RESUMEN:}

Las potencialidades pedagógicas que se pueden extraer de los dispositivos móviles en el ámbito educativo son numerosas. Por otra parte, los contenidos de Expresión Corporal de la materia de Educación Física son muy ricos para su tratamiento en el marco escolar y contribuir así en el desarrollo integral del alumnado. Este estudio expone una propuesta de innovación en ESO en la materia de Educación Física para trabajar los contenidos de Expresión Corporal, tradicionalmente discriminados por cierto profesorado de dicha disciplina, de una forma original e interdisciplinar apoyada en el uso del dispositivo móvil como herramienta educativa. En esta experiencia se ha evaluado la consecución de los objetivos didácticos planteados, el desarrollo de las competencias clave y el grado de satisfacción del alumnado con la propuesta planteada. Los resultados obtenidos indican que el aprendizaje móvil junto con la metodología empleada ha generado una mayor motivación intrínseca en el alumnado, ha favorecido la autonomía de trabajo, ha contribuido a la adquisición de aprendizajes significativos $\mathrm{y}$ ha invitado hacia una reflexión crítica sobre el uso responsable de dicho dispositivo. Asimismo, el alumnado quedó muy satisfecho con las actividades y tareas desarrolladas pertenecientes al bloque de Expresión Corporal.

PALABRAS CLAVE: EDUCACIÓN FÍSICA, APRENDIZAJE MÓVIL, EXPRESIÓN CORPORAL, EDUCACIÓN SECUNDARIA, TIC.

\section{ABSTRACT:}

The pedagogical potentials of mobile devices in education are numerous. Furthermore, the contents related to Body Expression, which are part of the curriculum for Physical Education, are very rich for their treatment in the schools and thus contribute to the integral development of the students. This research presents a proposal for innovation in Compulsory Secondary Education in the field of Physical Education to work on the contents related to Body Expression -traditionally discriminated against by some teachers of that discipline- in an original and interdisciplinary way supported by the use of the mobile device as an educational tool. In this experience, the achievement of the didactic objectives set, the development of the key competences and the degree of student satisfaction with the proposal have been evaluated. The results suggest that m-learning and the methodology used have generated a higher intrinsic motivation in students, have favored work autonomy, have contributed to the acquisition of meaningful learning and have led to a critical reflection on the responsible use of that device. Additionally, the students were very satisfied with the activities and tasks carried out belonging to the Body Expression block of contents.

KEYWORDS: PHYSICAL EDUCATION, MLEARNING, BODY EXPRESSION, SECONDARY SCHOOL, ICT. 


\section{INTRODUCCIÓN}

Resulta evidente que la presencia de ciertos dispositivos tecnológicos y las posibilidades que estos nos ofrecen están cobrando cada vez más relevancia en nuestra sociedad actual. El auge de las nuevas tecnologías y los continuos estudios científicos han ido demostrando que son herramientas que presentan múltiples posibilidades, tanto educativas como recreativas. $\mathrm{Y}$ es que, si revisamos algunos de los artículos bibliográficos más recientes sobre este tema, son muchos los autores que señalan sus aspectos beneficiosos y potenciales educativas, destacando la gran variedad de tipos de aprendizajes que se pueden adquirir a través de ellos, así como el propio componente motivador que llevan implícito y que facilita el proceso de enseñanza-aprendizaje.

La utilización de recursos TIC en el ámbito educativo, apoyada por una metodología basada en los modelos globalizadores y, concretamente, en el aprendizaje basado en proyectos, se convierten en dos elementos clave que se retroalimentan y caminan en la misma dirección para transmitir una serie de aprendizajes significativos que sean de utilidad para el contexto real en el que se desenvuelve el alumnado y, por tanto, proporcionar una educación de calidad ajustada a la realidad actual en la que vivimos.

Por otra parte, y en relación a la materia de Educación Física, cabe recalcar que el bloque de Expresión Corporal posee una riqueza de contenidos muy considerable con numerosas y destacables potencialidades pedagógicas. No obstante, dicho bloque ha sido tradicionalmente discriminado en la materia y el alumnado no suele ser muy receptivo a la impartición de este tipo de contenidos.

Por todo ello, los objetivos principales de este artículo se resumen en, por un lado, conocer y analizar la situación actual en relación al aprendizaje móvil en Educación Secundaria Obligatoria (ESO) así como sus ventajas y posibilidades educativas y, por otro, extraer unos resultados y conclusiones tras la aplicación de una propuesta de innovación en un centro de educación secundaria diseñada en base a lo expuesto anteriormente y a las necesidades e intereses del alumnado de dicho centro contribuyendo, de esta forma, a su formación integral y comprobando su grado de satisfacción con el contenido desarrollado en la propuesta. Todo ello hace referencia al trabajo final de máster llevado a cabo por Martínez (2017).

\section{MARCO CONCEPTUAL}

\subsection{El aprendizaje móvil. Aspectos generales y situación actual}

Atendiendo a la UNESCO (2013), el aprendizaje móvil conlleva el uso de tecnología móvil, ya sea sola o combinada con cualquier otro tipo de herramienta TIC con el propósito de facilitar el aprendizaje en un contexto determinado.

Siguiendo a López $(2017$, p. 3)

la tecnología móvil ha impactado de lleno en nuestras vidas y ha cambiado de forma sorprendente, no solo el modelo de acceso a la información y comunicación, sino también nuestra forma de vivir. Cada vez más, se consigue aumentar el potencial, la funcionalidad y la asequibilidad de estos dispositivos, y con ello se consiguen nuevas posibilidades de apoyar y favorecer el proceso de aprendizaje.

De hecho, si "damos una vuelta" por la bibliografía especializada sobre este tema, son numerosas las referencias que expresan las ventajas del uso de este tipo de dispositivos en el ámbito educativo recomendando su implantación $\mathrm{y}$ afirmando que son el futuro.

Por ejemplo, Cantillo, Roura y Sánchez (2012) afirman que

no debemos despreciar las facilidades tecnológicas que nos aporta un aparato de tan reducido tamaño destacando que los profesores y las profesoras acostumbrados a lidiar con aulas de informática o grandes pizarras digitales, conexiones inalámbricas y demás aplicaciones, a veces no son capaces de ver las posibilidades de un objeto tan al alcance de todos y tan conocido por todos. ( $\mathrm{p}$. 9)

Asimismo, en el Mobile World Congress en 2011 ya se destacaba que, tanto la tableta digital como el smartphone, se habían convertido en los dispositivos móviles de mayor impacto de los últimos años. Es indiscutible que sus características lo hacen una herramienta muy a tener en cuenta. De hecho, las características asociadas al aprendizaje móvil son (Cantillo et al, 2012, p 4):

- Portabilidad, debido al pequeño tamaño de los dispositivos.

- Inmediatez y conectividad mediante redes inalámbricas.

- Ubicuidad, ya que se libera el aprendizaje de barreras espaciales o temporales.

- Adaptabilidad de servicios, aplicaciones e interfaces a las necesidades del usuario. 
También existe la posibilidad de incluir accesorios como teclados o lápices para facilitar su uso.

En el Mobile World Congress de 2016 se expuso que en el siglo XXI los nuevos instrumentos de enseñanza en los centros educativos son los dispositivos móviles. Por lo tanto, el reto implica alcanzar que tanto el alumnado como el profesorado logren hacer un buen uso de estas herramientas para mejorar la calidad de la educación.

Sin embargo, a pesar de que las posibilidades de las TIC para el aprendizaje son de sobra conocidas, también podemos encontrar algunos impedimentos como, por ejemplo, la prohibición de algunas herramientas en las escuelas. $\mathrm{Y}$ es que la presencia de los dispositivos móviles en el aula está ocasionando la división de la comunidad educativa, pues mientras que unos son partidarios de su prohibición, otros comienzan a ver sus posibilidades pedagógicas. A pesar de ello, según estudios de la UNESCO (2013), las tecnologías móviles pueden ser un buen medio para mejorar el acceso, la equidad y la calidad de la educación en todo el mundo.

\subsection{Aprendizaje móvil en Secundaria. Papel del profesorado}

La llegada de las nuevas tecnologías ha conllevado ciertas variaciones en el rol del profesorado asumiendo un rol activo, pero muy distinto al que desarrollaba en años anteriores. Ahora es él la persona encargada de diseñar situaciones de aprendizaje, las cuales tienen la finalidad de formar de manera integral al estudiante; además, el docente se tiene que formar continuamente.

Por otra parte, nos encontramos que los estudiantes no son los mismos, ni las demandas de la sociedad son las que antes había. Se pueden encontrar diferentes etiquetas para describir a los jóvenes que actualmente estudian en los centros educativos como, por ejemplo, la generación de Google, la generación de la red o los nativos digitales. Siguiendo a Gibbons (2007), todos estos términos se utilizan para resaltar la importancia de las nuevas tecnologías en la vida de los jóvenes. No obstante, el simple hecho de haber nacido en esta generación no implica una mayor preparación o predisposición ante las nuevas tecnologías. Según Helsper y Enyon (2009), la experiencia, la amplitud de uso, la autoeficacia y la educación son incluso más importantes que la edad para explicar cómo las personas se "convierten" en nativos digitales. Igualmente, Kirschner y Bruyckere (2017) presentan pruebas científicas que demuestran que no hay tal cosa como un nativo digital que es informatizado simplemente porque nunca ha conocido un mundo que no era digital.

Así pues, los centros educativos deben partir de las experiencias de su alumnado y enriquecerse de estos conocimientos para poder extraer el máximo potencial de las tecnologías $\mathrm{y}$, por lo tanto, aplicarlas en los procesos de enseñanza-aprendizaje.

\subsection{Experiencias en Secundaria}

Antes de comenzar a hacer referencia de forma concreta a algunos de los estudios revisados sobre experiencias del aprendizaje móvil en ESO, conviene destacar que muchos de ellos coinciden en que una de las ventajas que otorga el uso de este tipo de herramientas en el ámbito educativo es la motivación que consigue extraer en el alumnado. Sin excedernos con respecto a este término, sí que es preciso destacar que la motivación es un concepto que ha sido objeto de estudio durante mucho tiempo debido al papel principal que ocupa dentro de la psicología como fuerza activadora de la conducta humana.

De hecho, algunos de los estudios revisados sobre el aprendizaje móvil en secundaria hacen directamente referencia a una de las teorías más famosas en relación con la motivación: la Teoría de la Autodeterminación de Deci y Ryan (2000).

Un ejemplo de estudio sobre el aprendizaje móvil basado en la teoría de la autodeterminación es el llevado a cabo por Nikou y Economides (2016). En dicho estudio se realizó una experiencia basada en la evaluación a través de los dispositivos móviles concluyendo, entre otras cuestiones, la importancia de los factores motivacionales para la integración de este tipo de tecnologías, así como la satisfacción de las tres necesidades psicológicas a las que hace referencia la teoría de la autodeterminación.

Ibañez, Vicent y Brouard (2012) evaluaron un programa basado en el aprendizaje móvil para el nivel de secundaria, cuyos resultados concluyeron que la adquisición de contenidos fue positiva y que el factor motivacional proporcionado por los dispositivos móviles es alto. Por otra parte, en dicho estudio no se pudo determinar si estos dispositivos pueden suponer una distracción para el alumnado.

En definitiva, resultan evidentes los aspectos positivos que podemos extraer del aprendizaje móvil en ESO, ya no únicamente por la motivación que suele generar en el alumnado, sino también por las posibilidades a nivel didáctico que nos ofrece para impartir diversidad de contenidos. No obstante, y además de las posibles distracciones que pueden generar en diversas ocasiones, también debemos tener presente que el simple hecho de utilizar este 
tipo de herramientas no garantiza el éxito de una propuesta didáctica determinada, por lo que resulta fundamental que el uso de este elemento esté acompañado por un planteamiento didáctico adaptado a las necesidades e intereses del alumnado.

\subsection{Educación Física y Expresión Corporal}

La materia de Educación Física y, concretamente, el bloque de contenidos que hace referencia a la Expresión Corporal, reúne unas características que favorecen la adquisición de las competencias para la vida. Atendiendo al Decreto 87/2015 en su artículo 2, la Educación Física, mediante el desarrollo de la competencia motriz, establece la integración de los conceptos, procedimientos y actitudes vinculados al cuerpo, al movimiento y su relación con el entorno. No obstante, el enfoque de esta materia no solo hace referencia al desarrollo motriz, sino también a la adquisición de todas aquellas conductas que mejoran la salud y, con ello, el bienestar físico, psíquico y emocional de los alumnos como personas integrantes de una sociedad de bienestar. Asimismo, la materia de Educación Física contribuye al desarrollo de todas y cada una de las competencias clave que constan en el currículo oficial, contribuyendo al desarrollo de la competencia digital (la más relacionada con la temática del presente artículo) mediante diferentes propuestas donde los estudiantes deben usar y aplicar de forma correcta las nuevas tecnológicas para explorar, investigar, desarrollar y exponer diversos temas relacionados con los contenidos de dicha materia.

En relación a las actividades de Expresión Corporal y de nuevo siguiendo lo expuesto en el Decreto 87/2015 en su artículo 2, cabe destacar que estas "favorecen la desinhibición y la exteriorización de sentimientos y emociones al mismo tiempo que se consolidan como elementos básicos de la comunicación, la relación con los demás y la expresión”. Dentro de este bloque y para esta etapa educativa se estimula la creatividad del alumnado en relación al montaje de danzas, coreografías sencillas u otros trabajos de expresión, en primer lugar desde una perspectiva de colaboración y, en segundo lugar, aumentando la complejidad de las tareas hacia una vertiente más participativa y relacionada con la toma de decisiones desde un espíritu más creativo y emprendedor. Todo ello, sumado a los aspectos positivos ya mencionados sobre el aprendizaje móvil, nos va a permitir transmitir una serie de aprendizajes significativos que contribuyan al correcto desarrollo del alumnado en cuestión.
Sin embargo y a pesar de la riqueza que poseen este tipo de contenidos para ser aplicados en el ámbito educativo, la Expresión Corporal ha estado tradicionalmente algo discriminada dentro de la materia de Educación Física, siendo numerosos los autores que van en esta línea. Por ejemplo, Coterón y Sánchez (2012) no dudan en remarcar que, sin temor a equivocarse, se puede decir que la Expresión Corporal viaja desde 1990 en la maleta de la Educación Física como un polizón en un barco. Esto al final también se transmite al alumnado, el cual suele ser menos receptivo en relación a la impartición de este tipo de contenidos frente a otros más habituales como los deportes.

Precisamente, de lo expresado en este último párrafo surge una de las razones por las que se ha creído conveniente llevar a cabo una propuesta de innovación como la que se expone en los siguientes apartados.

\section{MÉTODO}

\subsection{Contexto, participantes y justificación del estudio}

La propuesta de innovación está diseñada para el nivel de $4^{\circ}$ de la ESO del Instituto Número 1 de Jávea, centro de titularidad pública dependiente de la Consellería de Educación y capacitado para la prestación de ESO y Bachillerato. El alumnado participante corresponde a los diferentes cursos del nivel $4^{\circ}$ ESO de dicho centro educativo, teniendo una edad de entre 15 y 17 años. Estos participantes han sido elegidos principalmente por dos razones: por un lado, por ser el investigador el docente de la totalidad de cursos correspondientes al nivel de $4^{\circ}$ ESO, teniendo, de esta forma, el acceso y la disposición necesaria para desarrollar todo lo planificado, así como un mayor número de participantes. Por otro, por ser sujetos cuyas características encajan perfectamente con la propuesta desarrollada, haciendo referencia especialmente a los contenidos a tratar, así como a los objetivos definidos en el propio trabajo.

Así pues, los participantes del presente estudio se resumen en la siguiente tabla:

Tabla 1. Participantes del estudio

\begin{tabular}{ccccc}
\hline & $\mathbf{4}^{\mathbf{}} \mathbf{A}$ & $\mathbf{4}^{\mathbf{}} \mathbf{B}$ & $\mathbf{4}^{\mathbf{0}} \mathbf{C}$ & Total \\
\hline $\mathbf{N}^{\mathbf{0}}$ participantes & 24 & 21 & 24 & 69 \\
\hline
\end{tabular}

En relación a la justificación del presente estudio, se resume en: 
- El hecho de no encontrar estudios relacionados con el aprendizaje móvil y la materia de Educación Física para la etapa educativa de ESO.

- Las necesidades contextuales del alumnado, destacando la detección de un mal uso de los dispositivos móviles dentro del contexto escolar.

- Las ventajas a nivel didáctico que nos pueden ofrecer los dispositivos móviles como herramienta educativa.

- La posibilidad de aprovechar la multitud de enseñanzas y aprendizajes que se pueden transmitir con el tratamiento de contenidos de Expresión Corporal combinados con una metodología innovadora alejándonos de la discriminación que sufre, en ocasiones, este contenido y tratando de satisfacer al alumnado con el mismo.

\subsection{Objetivos}

Los objetivos principales que se pretenden alcanzar son:

- Conocer la situación actual del aprendizaje móvil como recurso TIC en la ESO y, de forma más específica, con respecto a la materia de Educación Física.

- Diseñar y aplicar una propuesta de intervención en un centro de educación secundaria liderado desde la materia de Educación Física con el propósito de atender a las necesidades e intereses del alumnado para contribuir en su desarrollo integral.

- Comprobar el grado de satisfacción del alumnado con respecto al tratamiento de los contenidos de Expresión Corporal de una forma innovadora como la planteada en la propuesta de intervención.

\subsection{Metodología}

En el presente estudio se ha sido fiel a las nuevas tendencias educativas centradas en el desarrollo personal del alumnado y todo ello atendiendo a autores de referencia como Zabala y Arnau (2014). En base a ello, se ha concretado un método de actuación que, básicamente, con su seguimiento y análisis nos permitirá saber si el alumnado ha conseguido alcanzar los objetivos didácticos establecidos en la propuesta de innovación y, de forma derivada, conocer en qué medida se ha alcanzado uno de los objetivos concretados para el presente estudio. Y es que no debemos olvidar que, con la investigación educativa, entre otras cosas, se busca dar una solución a un problema determinado englobado en un contexto educativo concreto. De hecho, son varios los autores que siguen esta línea como, por ejemplo, Hérnandez (2001) el cual remarca la importancia de que en la investigación educativa no únicamente se debe tener como objetivo el estudio de métodos, procedimientos y técnicas utilizadas para obtener un conocimiento, sino también debe aplicarse para solucionar los problemas educativos y sociales.

En definitiva, partiendo de todo lo expuesto en el marco teórico y los párrafos anteriores, se puede concretar que el presente estudio es de carácter inductivo, partiendo de un problema real al cual se ajusta el método y diseño de las fases para atender a las necesidades contextuales del alumnado.

\subsection{Fases y temporalización del estudio.}

A continuación, se muestra una tabla resumen que hace referencia a las fases y temporalización del proceso de estudio:

Tabla 2. Temporalización del proceso de estudio

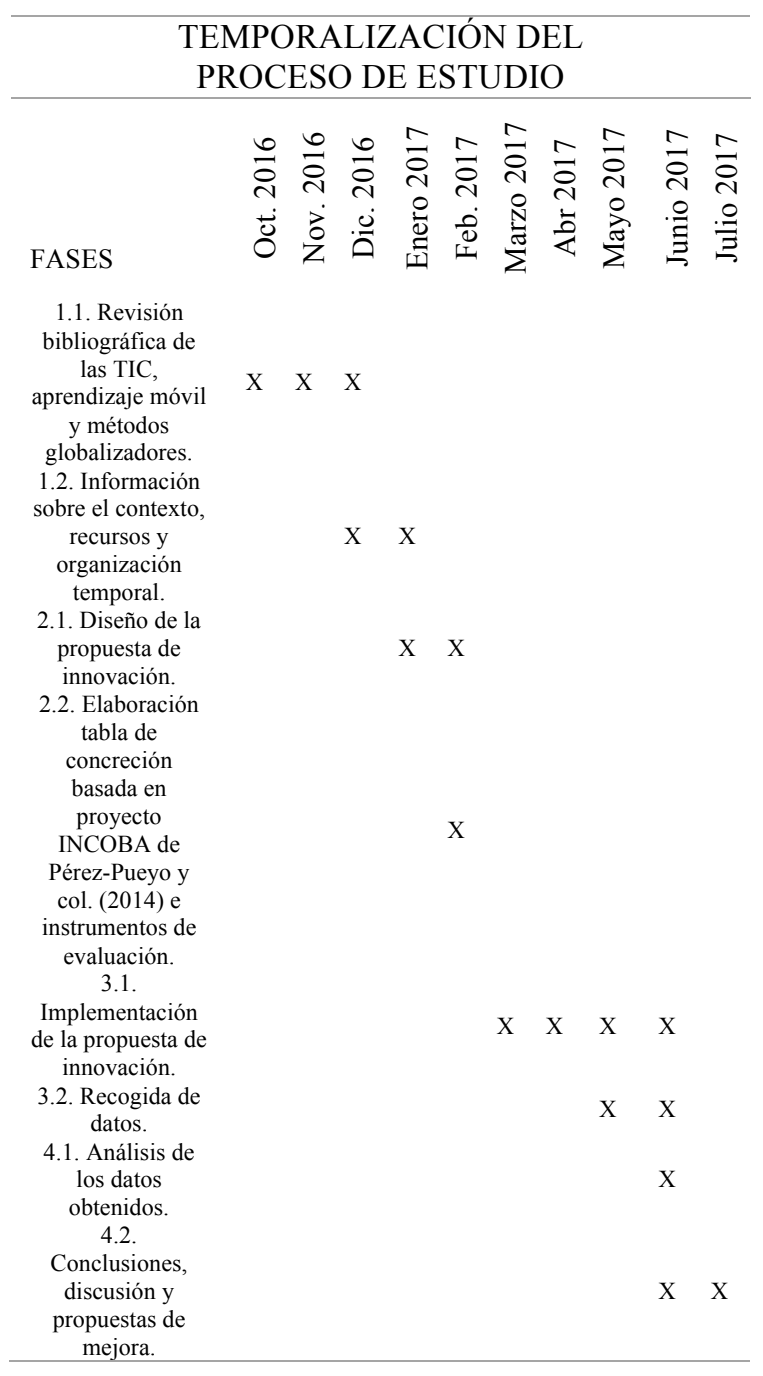




\subsection{Temporalización, tareas y actividades previstas}

En la siguiente tabla se expone, de manera resumida y gráfica, la temporalización de la propuesta de innovación, las tareas en las que giran las propias fases de la misma, las actividades a llevar a cabo y las materias que participan en la realización de las diversas actividades programadas:

Tabla 3. Temporalización, tareas, actividades y materias implicadas. Fuente: elaboración propia.

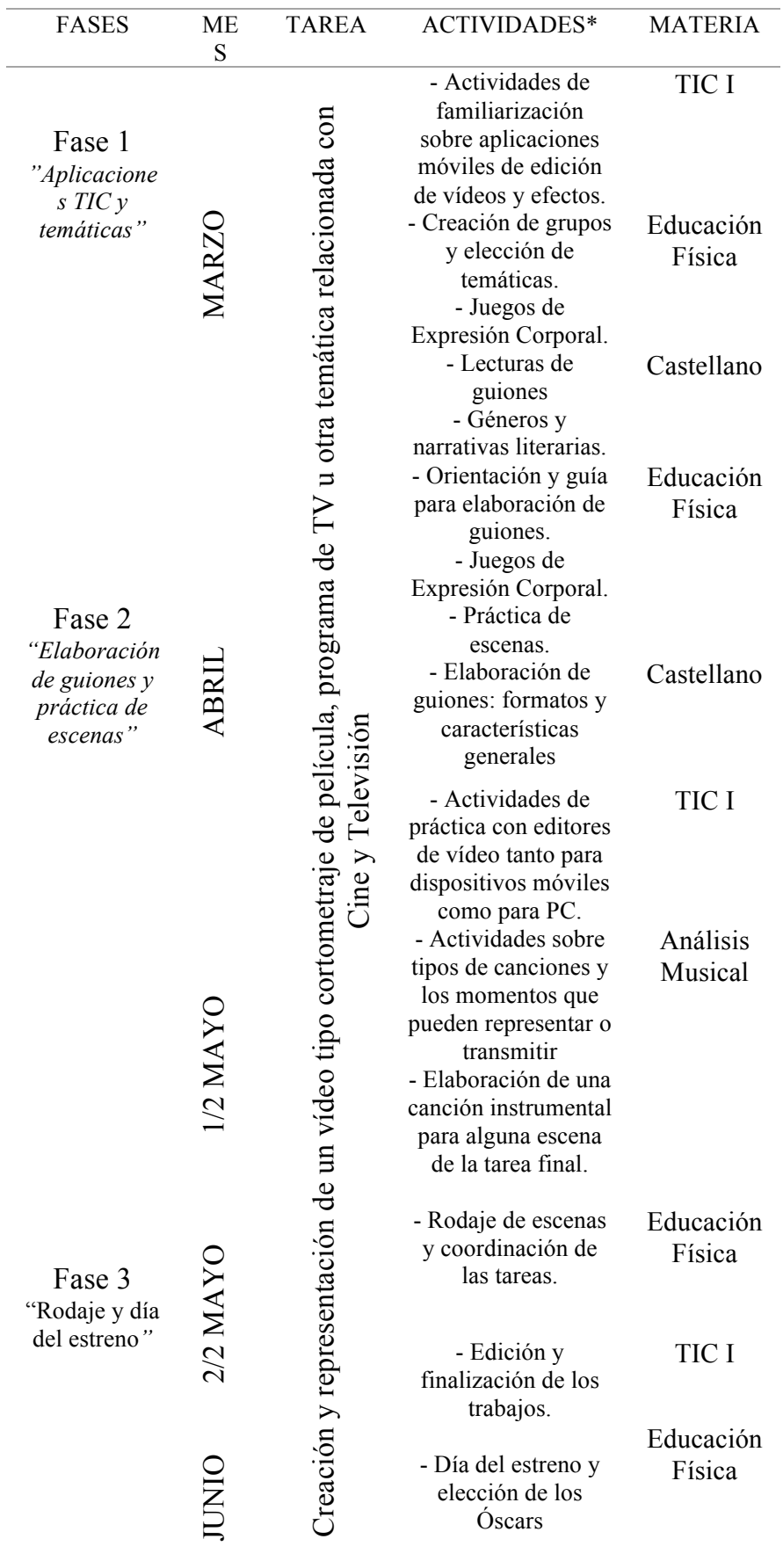

*Actividades secuenciadas según el orden de aparición. Algunas de ellas comparten espacios temporales durante su desarrollo.

\section{RESULTADOS}

En este capítulo se van a exponer los resultados recogidos de los instrumentos de evaluación diseñados y que aparecen reflejados con detalle en el trabajo fin de máster de Martínez (2017).

\subsection{Datos recogidos en el cuestionario previo}

Antes de exponer los resultados de las diferentes preguntas que forman el cuestionario previo al comienzo de la propuesta, es preciso concretar que de los 69 participantes del estudio, 52 de ellos rellenaron y entregaron el cuestionario bien vía Edmodo o vía papel. El resto de estudiantes no entregaron el cuestionario en las fechas indicadas.

A continuación, se exponen los gráficos resumen que reflejan las preguntas del cuestionario y las contestaciones del alumnado objeto de estudio:

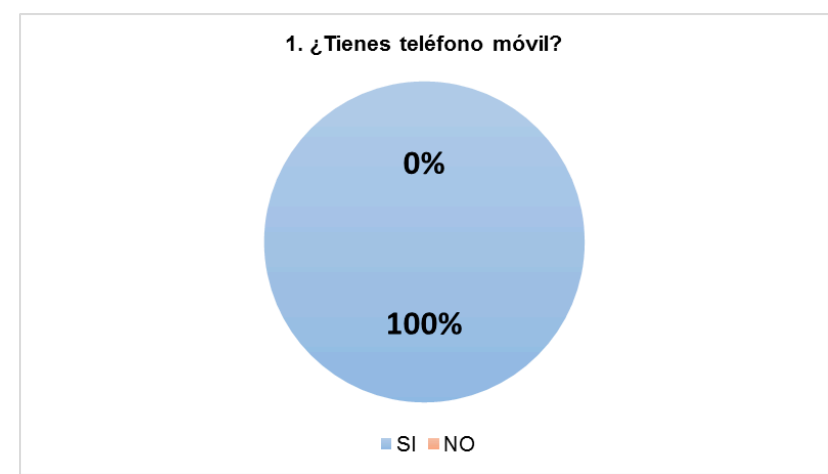

Gráfico 1. Resultado de la pregunta 1 del cuestionario previo dirigido al alumnado participante del nivel $4^{\circ}$ ESO

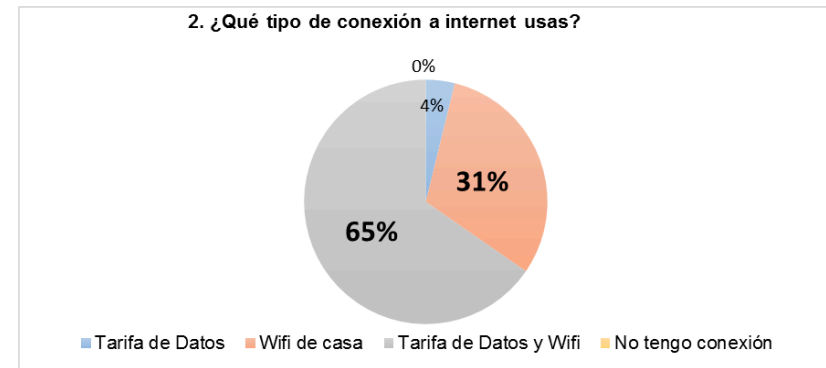

Gráfico 2. Resultado de la pregunta 2 del cuestionario previo dirigido al alumnado participante del nivel $4^{\circ} \mathrm{ESO}$ 


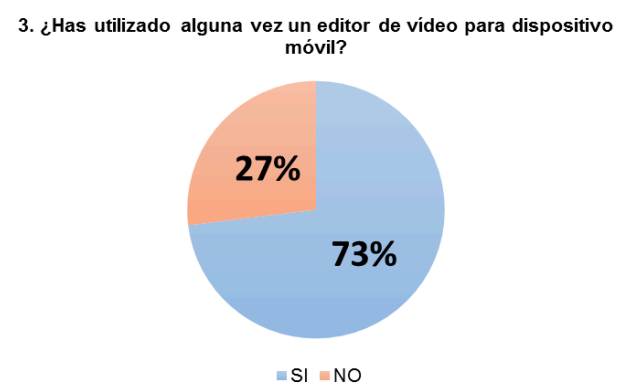

Gráfico 3. Resultado de la pregunta 3 del cuestionario previo dirigido al alumnado participante del nivel $4^{\circ}$ ESO.

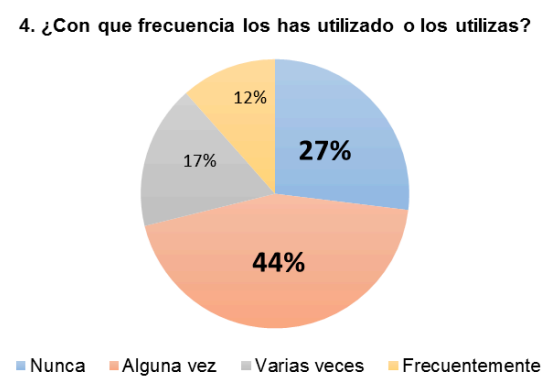

Gráfico 4. Resultado de la pregunta 4 del cuestionario previo dirigido al alumnado participante del nivel $4^{\circ}$ ESO.

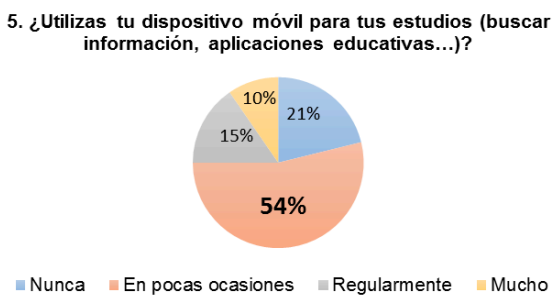

Gráfico 5. Resultado de la pregunta 5 del cuestionario previo dirigido al alumnado participante del nivel $4^{\circ}$ ESO.

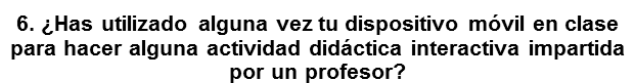
por un profesor?

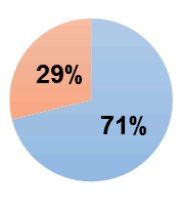

$\llbracket \mathrm{SI} \bowtie \mathrm{NO}$

Gráfico 6. Resultado de la pregunta 6 del cuestionario previo dirigido al alumnado participante del nivel $4^{\circ}$ ESO.
7. ¿Te han demandado en alguna ocasión la entrega de un trabajo en formato vídeo?

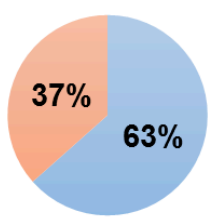

$\varpi \mathrm{SI} \equiv \mathrm{NO}$

Gráfico 7. Resultado de la pregunta 7 del cuestionario previo dirigido al alumnado participante del nivel $4^{\circ}$ ESO.

\subsection{Datos relativos a la encuesta final}

En este apartado se muestran los datos obtenidos de la encuesta final realizada a través de una de las funcionalidades de la plataforma educativa Edmodo. Se trata de una encuesta muy simple con el objetivo de medir el grado de satisfacción del alumnado con el contenido trabajado, así como con la metodología innovadora con la que se ha impartido. Esta encuesta se ha enviado vía Edmodo, por lo que directamente se expone una captura de pantalla de la encuesta con los resultados.

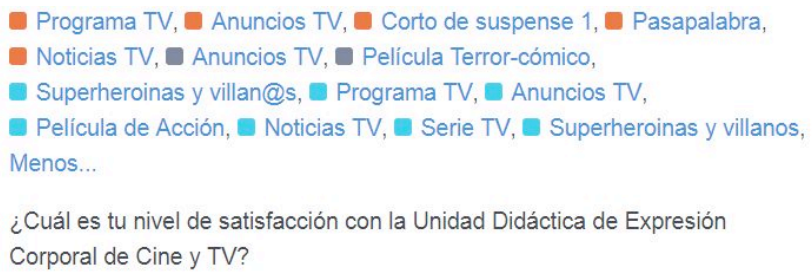

Poco satisfecho. Hubiera preferido hacer una coreografía en directo y dejarme de tanto rodaje y edición de vídeo con el móvil y/o PC. 0 voto(s)

Regular. La tarea ha estado bien pero nos ha sido difícil expresarnos y utilizar las aplicaciones móviles y/o de PC para editar los vídeos. EI "Día del Estreno" no me ha gustado mucho... 0 voto(s)

Satisfecho. La tarea ha sido muy divertida y motivante, nos hemos divertido en el rodaje y con la ayuda de la edición de vídeo nos ha quedado un trabajo muy bueno. Trabajar la expresión corporal de esta forma me ha gustado bastante. $29 \%, 9$ voto(s)

Muy satisfecho. Nos hemos divertido mucho en el rodaje, hemos interpretado muy bien y con las app de móvil y/o PC nos ha quedado una producción digna de Hollywood. Me ha encantado trabajar la expresión corporal de esta forma y el "Día del Estreno" con la entrega de los "Óscar" me ha parecido muy divertido y motivante. $71 \%, 22$ voto(s)

Votos totales: 31 (Refrescar)

Imagen 1. Captura de pantalla Edmodo de los resultados de la encuesta final cumplimentada por el alumnado participante de $4^{\circ} \mathrm{ESO}$. 
En primer lugar, cabe destacar que la participación en esta encuesta ha sido baja. Únicamente 31 estudiantes del total de 69 han completado la encuesta vía Edmodo. No obstante, y a pesar de que la participación no haya sido la ideal, de los 31 estudiantes que han completado la encuesta, un $71 \%$ ha marcado la respuesta de máxima satisfacción con la propuesta innovadora realizada y un $29 \%$ ha votado "Satisfecho", siendo esta la segunda respuesta más positiva de las 5 posibles. Por tanto, consideramos muy positivo que ni un solo estudiante haya votado alguna de las opciones referidas a una menor satisfacción con la propuesta llevada a la práctica.

\subsection{Rúbrica de evaluación de la tarea competencial}

El proceso de concreción curricular basado en el proyecto INCOBA, así como la rúbrica para la evaluación de la tarea competencial, han llevado un proceso de elaboración muy complejo que queda detallado en el trabajo final de máster de Martínez (2017) al que se hace referencia directa en el presente artículo. Por ello, se aconseja la consulta del mismo si se pretende conocer más detalles de dicho proceso.

Esta rúbrica junto al proceso de concreción que la sustenta es la herramienta principal que permite valorar la consecución de los objetivos didácticos de la propuesta de innovación y, de forma derivada, el segundo objetivo concretado en el presente estudio.

A continuación, se exponen los resultados obtenidos:
Tabla 4. Datos recogidos de la rúbrica de evaluación

\begin{tabular}{|c|c|c|c|c|c|}
\hline \multirow[b]{2}{*}{$\begin{array}{l}\text { Aspectos } \\
\text { evaluados en la } \\
\text { rúbrica }\end{array}$} & \multicolumn{4}{|c|}{$\begin{array}{l}\mathrm{N}^{\mathrm{o}} \text { de alumnos que han } \\
\text { recibido la calificación } \\
\text { correspondiente. }\end{array}$} & \multirow[b]{2}{*}{$\begin{array}{l}\text { Nota } \\
\text { Media }\end{array}$} \\
\hline & 0 & 1 & 2 & 3 & \\
\hline $\begin{array}{l}\text { Adecuación con el } \\
\text { guion/temática } \\
\text { asignada. }\end{array}$ & 5 & 0 & 0 & 64 & 2.77 \\
\hline $\begin{array}{c}\text { Exposición del } \\
\text { argumento/tema. }\end{array}$ & 5 & 5 & 21 & 38 & 2.32 \\
\hline $\begin{array}{c}\text { Lenguaje y } \\
\text { postura corporal. } \\
\text { Papel de } \\
\text { actores/actrices }\end{array}$ & 5 & 0 & 37 & 27 & 2.25 \\
\hline $\begin{array}{l}\text { Volumen, } \\
\text { entonación y } \\
\text { velocidad. }\end{array}$ & 5 & 5 & 36 & 23 & 2.12 \\
\hline Vestuario. & 5 & 5 & 38 & 21 & 2.08 \\
\hline $\begin{array}{c}\text { Producción. Uso } \\
\text { de las TIC en el } \\
\text { montaje y el } \\
\text { rodaje de la } \\
\text { tarea. }\end{array}$ & 5 & 5 & 31 & 28 & 2.18 \\
\hline Trabajo en grupo. & 5 & 9 & 16 & 39 & 2.29 \\
\hline Tiempo del vídeo. & 5 & 9 & 0 & 55 & 2.52 \\
\hline Originalidad. & 5 & 5 & 19 & 40 & 2.36 \\
\hline $\begin{array}{l}\text { Lugares de } \\
\text { rodaje. }\end{array}$ & 10 & 4 & 28 & 27 & 2.05 \\
\hline $0=$ Incompleto & $-\mathrm{J}$ & & Q. & - & lente \\
\hline
\end{tabular}

Nota media por apartado sobre el máximo de 3 puntos.

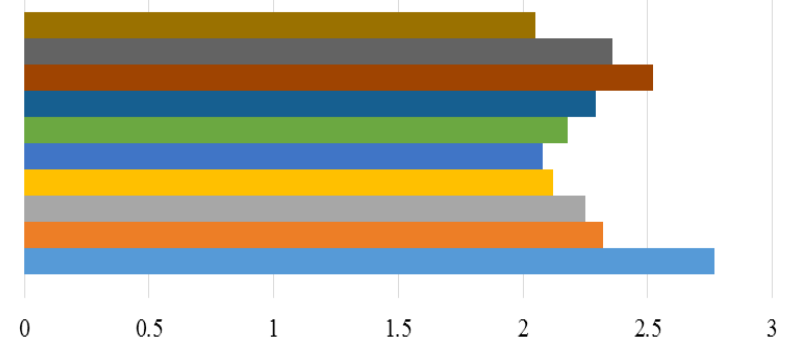

-Lugares de rodaje

- Originalidad

- Tiempo del vídeo

- Trabajo en grupo

- Producción. Uso de las TIC en el montaje y el rodaje

- Vestuario

-Volumen, entonación y velocidad

-Lenguaje y postura corporal. Papel de actores

-Exposición del aroumento/tema

- Adecuación con el guion/temática asignada

Gráfico 8. Resultados finales por apartado de la rúbrica de evaluación de la tarea. 


\section{DISCUSIÓN Y CONCLUSIONES}

Tras la recogida $\mathrm{y}$ análisis de todos los datos expuestos en el punto anterior, es necesario mostrar las conclusiones más relevantes con respecto a los diferentes objetivos concretados en el presente estudio. Es por ello que, a continuación, se van a clasificar dichas conclusiones de manera organizada y resumida según los objetivos planteados:

Tabla 5. Síntesis de los elementos referentes al objetivo 1

\section{OBJETIVO 1}

Conocer la situación actual del aprendizaje móvil como recurso TIC en la Educación Secundaria Obligatoria y, de forma más específica, con respecto a la materia de Educación Física.

\section{NECESIDADES}

- Falta de conocimiento sobre las posibilidades del dispositivo móvil como herramienta TIC en el ámbito educativo.

- Ignorancia sobre la existencia de experiencias en ESO tanto en la materia de Educación Física como en otras de la misma etapa educativa.

\section{CONCLUSIONES}

- Las herramientas TIC pueden enriquecer los procesos de enseñanza-aprendizaje.

- El dispositivo móvil ofrece multitud de ventajas para ser utilizado en el ámbito educativo destacando:

- Su reducido tamaño.

- Facilidad y rapidez de conexión a redes inalámbricas.

- Numerosas aplicaciones de las que se puede hacer uso.

- Forma parte de nuestra sociedad, especialmente entre los jóvenes.

- Existe una división en la comunidad educativa entre los que defienden la prohibición en los centros del teléfono móvil frente a aquellos que abogan por una educación responsable del mismo.

- El docente debe formarse continuamente.

- La creación de políticas innovadoras dando soporte a la formación del profesorado sería muy positiva para la causa.

- Las experiencias en ESO corroboran las posibilidades educativas del aprendizaje móvil y destacan la motivación intrínseca que generan en el alumnado.

- El uso del dispositivo móvil puede generar, en ocasiones, distracciones entre el alumnado.

- El docente debe conocer las características de las aplicaciones móviles para emplear aquellas adecuadas según su propuesta didáctica y sustentado por una buena metodología adaptada al contexto correspondiente.

- Escasos estudios en ESO referentes a la materia de Educación Física.
Tabla 6. Síntesis de los elementos referentes al objetivo 2.

\section{OBJETIVO 2}

Diseñar y aplicar una propuesta de intervención en un centro de educación secundaria liderado desde la materia

de Educación Física con el propósito de atender a las necesidades e intereses del alumnado para contribuir en su desarrollo integral.

\section{NECESIDADES}

- Mal uso de los dispositivos móviles por parte de algunos estudiantes del IES Número 1 de Jávea.

- Dificultad general por parte del alumnado a la hora de desarrollar actividades relacionadas con contenidos de Expresión Corporal.

INSTRUMENTOS / MEDIOS RECOGIDA INFORMACIÓN

- Cuestionario uso del dispositivo móvil. - Rúbrica evaluación tarea final.

- App "Cuaderno del Profesor".

\section{CONCLUSIONES}

- La propuesta ha resultado muy válida para trabajar los contenidos de Expresión Corporal y contribuir al desarrollo integral del alumnado participante.

- El aprendizaje móvil ha conllevado una mayor motivación por parte del alumnado en la elaboración de la tarea y ha contribuido a reflexionar de una manera crítica en cuanto a un uso responsable y productivo del mismo.

- La metodología empleada ha favorecido la autonomía de trabajo, aprender a aprender y la adquisición de aprendizajes significativos.

Tabla 7. Síntesis de los elementos referentes al objetivo 3

$$
\text { OBJETIVO } 3
$$

Comprobar el grado de satisfacción del alumnado con respecto al tratamiento de los contenidos de Expresión

Corporal de una forma innovadora como la planteada en la propuesta de intervención. NECESIDADES

- Poca predisposición general por parte del alumnado de ESO a practicar actividades referentes al bloque de Expresión Corporal.

\section{INSTRUMENTOS / MEDIOS RECOGIDA} INFORMACIÓN

- Encuesta de satisfacción vía Edmodo. CONCLUSIONES

- Alumnado satisfecho o muy satisfecho con la propuesta desarrollada de Expresión Corporal apoyada en el aprendizaje móvil.

En relación a las limitaciones del estudio, en primer lugar, cabe destacar que el carácter interdisciplinar de la propuesta diseñada no se ha cumplido según lo establecido. La planificación hacía referencia a que cada materia participaba en la propuesta a través de una serie de actividades. No 
obstante, dada la condición de interinidad del investigador en el IES Número 1 de Jávea, no era viable cumplir el diseño tal cual estaba planificado y fue el propio investigador quien dedicaba tiempo extra a aquellos estudiantes que requerían de una cierta ayuda en cuanto a aspectos más relativos a otras materias.

Por otra parte, otras de las limitaciones van relacionadas con el factor tiempo. Haciendo referencia a los instrumentos de recogida de datos, la encuesta de satisfacción cumplimentada vía Edmodo al finalizar la propuesta fue completada por menos de la mitad de los participantes del estudio. Este hecho se debe principalmente a que dicha encuesta se envió una vez finalizadas las clases presenciales, en fechas donde el alumnado suele desconectar por encontrarse en periodo vacacional. Así pues, la falta de tiempo y otra serie de circunstancias dio lugar a no tener otra opción en cuanto al envío de dicha encuesta en esas fechas, lo que ha conllevado una participación por debajo del $50 \%$ del total de participantes.

Para terminar con lo referente a los instrumentos de evaluación, otra limitación que se puede destacar es que ninguno de ellos fue sometido a un juicio de expertos, lo que podría haber aumentado la calidad de los mismos incrementando su fiabilidad y validez. Sin embargo, cabe destacar a favor que, a pesar de lo comentado anteriormente, el investigador los ha tratado de diseñar con el máximo rigor y apoyado en bibliografía especializada citada en sus apartados correspondientes.

Por último, y en cuanto a las futuras líneas de actuación, el primer aspecto a destacar es la escasez de investigaciones que relacionen la Educación Física con el aprendizaje móvil en esta etapa educativa. Con lo cual, no cabe ninguna duda de que un camino a explorar es la investigación y la puesta en práctica de propuestas innovadoras referentes a la materia de Educación Física que se apoyen en el aprendizaje móvil como herramienta para aprovechar sus posibilidades en la multitud de contenidos que se pueden impartir en dicho ámbito.

Por otra parte, tampoco se encontró en la bibliografía estudios o experiencias que trataran contenidos de Expresión Corporal mediante trabajos basados en el cine y la televisión. Así pues, sería muy interesante profundizar en la riqueza de contenidos que se pueden impartir en este bloque para plantearlos a través de propuestas innovadoras que se alejen de las tradicionales y que encajen mejor con las necesidades e intereses que tienen los estudiantes en la actualidad.

\section{REFERENCIAS}

Cantillo, C., Roura, M. y Sánchez, A. (2012). Tendencias actuales en el uso de dispositivos móviles en educación. La educación Digital Magazine, 147, 120.

Recuperado de http://educoas.org/portal/la_educacio n_digital/147/pdf/ART_UNNED_EN.pdf

Coterón, J. y Sánchez, G. (2012). Expresión Corporal en Educación Física: la construcción de una disciplina. EmásF. Revista Digital de Educación Física (14), 164-175.

Deci, E. L. y Ryan, R. M. (2000). The what and why of goal pursuits: Human needs and the self-determination of behavior. Psychological Inquiry, 11, 227-268.

Decreto $87 / 2015$, de 5 de junio, del Consell, por el que establece el currículo y desarrolla la ordenación general de la Educación Secundaria Obligatoria y del Bachillerato en la Comunitat Valenciana. DOGV, $n^{\circ} 7544,2015$, 10 junio.

Gibbons, S. (2007). Redefining the roles of Information professionals in Higher education to engage the net generation. Paper presented at EDUCAUSE, Australasia. Recuperado de http://www.caudit.edu.a u/educauseaustralasia07/authors_papers/Gibbons2.p df

Helsper, E. y Eynon, R. (2009) Digital natives: where is the evidence? British educational research journal, 36(3), 503-520.

Hernández, F. (2001). Bases metodológicas de la investigación educativa. I fundamentos (ed. 2). Murcia: Diego Marín.

Ibañez, A., Vicent, N. y Asensio, M. (2012). Aprendizaje informal, patrimonio y dispositivos móviles. Evaluación de una experiencia en educación secundaria. Didáctica de las Ciencias Experimentales y Sociales, 26, 3-18.

Kirschner, P. A. y Bruyckere, P. D. (2017). The myths of the digital native and the multitasker. Teaching and Teacher Education, 67, 135-142.

López, B. (2017). Utilización del vídeo como recurso TIC para introducir la cultura murciana en las AAHH. Revista DIM, 35, 1-10

Martínez, A. (2017). Aprendizaje móvil en Educación Física. Una propuesta de innovación en ESO. (TFM).

Mobile World Congress. (2015). Profesores, escuelas y expertos en educación: la acción se pasa al Mobile World Congress. Recuperado de http://mobileworldcapital. com/es/profesores-escuelas-y-expertos-eneducacion-la-accion-se-pasa-al-mobile-worldcongress/

Nikou, S. A, y Economides, A. A. (2016). The impact of paperbased, computer-based and mobile-based selfassessment on students' science motivation and achievement. Computers in Human Behavior, 55, $1241-1248$

Pérez-Pueyo, A. (coord.). (2014). Programar y evaluar competencias básicas en 15 pasos. Barcelona: GRAO.

UNESCO (2013). El futuro del aprendizaje móvil. Implicaciones para la planificación y la formulación de politicas. Publicado por la Organización de las Naciones Unidas para la Educación, la Ciencia y la Cultura.

Recuperado de http://unesdoc.unesco.org/images/00 21/002196/219637s.pdf 
Zabala, A. y Arnau, L. (2014). Métodos para la enseñanza de las competencias. Barcelona: GRAO. 